\title{
Functional Organotypic Cultures of Prostate Tissues
}

\section{A Relevant Preclinical Model that Preserves Hypoxia Sensitivity and Calcium Signaling}

Sandy Figiel, ${ }^{*}$ Côme Pasqualin, ${ }^{\dagger}$ Fanny Bery, ${ }^{*}$ Veronique Maupoil, ${ }^{\dagger}$ Christophe Vandier, ${ }^{*}$ Marie Potier-Cartereau, ${ }^{*}$ Isabelle Domingo, ${ }^{*}$ Roseline Guibon, ${ }^{\star \ddagger}$ Franck Bruyere, ${ }^{\S}$ Karine Maheo, ${ }^{*}$ and Gaelle Fromont* ${ }^{\star \ddagger}$

From Inserm UMR U1069, * Tours; the EA 7349 Universite de Tours, Tours ${ }^{\dagger}$; the Department of Pathology, ${ }^{\ddagger}$ and the Department of Urology, ${ }^{\S}$ CHU-Universite de Tours, Tours, France

Accepted for publication February 21, 2019.

Address correspondence to Gaelle Fromont, M.D., Ph.D., Service d'Anatomie Pathologique, Hopital Bretonneau, CHRU Tours, Blvd. Tonnelle, 37000 Tours, France. E-mail: gaelle.fromont-hankard@univtours.fr.

\begin{abstract}
In prostate cancer research, there is a lack of valuable preclinical models. Tumor cell heterogeneity and sensitivity to microenvironment signals, such as hypoxia or extracellular calcium concentration, are difficult to reproduce. Here, we developed and characterized an ex vivo tissue culture model preserving these properties. Prostate tissue slices from 26 patients were maintained ex vivo under optimized culture conditions. The expression of markers associated with proliferation, androgen-receptor signaling, and hypoxia was assessed by immunostaining. A macroscope was used to achieve realtime calcium fluorescence optical imaging. Tissue morphology was maintained successfully without necrosis for 5 days. Compared with native tumors and tissue cultured with androgens, androgen deprivation in the medium led to decreased expression of both androgen receptor and its target gene products, prostate specific antigen (PSA) and ETS-related gene (ERG). Ex vivo cultured slices also were sensitive to hypoxia because carbonic anhydrase IX and zinc finger E-box binding homeobox 1 (Zeb1) protein levels increased in $1 \%$ oxygen. Exposure of slices to supraphysiological extracellular $\mathrm{Ca}^{2+}$ concentration induced a robust and rapid $\mathrm{Ca}^{2+}$ entry, with a greater response in tumor compared with nontumor tissue. This ex vivo model reproduces the morphologic and functional characteristics of human prostate cancer, including sensitivity to androgen deprivation and induced response to hypoxia and extracellular $\mathrm{Ca}^{2+}$. It therefore could become an attractive tool for drug response prediction studies. (Am J Pathol 2019, 189: 1268-1275; https://doi.org/10.1016/j.ajpath.2019.02.017)
\end{abstract}

Although prostate cancer ( $\mathrm{PCa}$ ) is the second leading cause of cancer-related death among men in developed countries, the transfer of scientific findings into medical progress is challenged by the lack of valuable preclinical models. Immortalized PCa cell lines have acquired several mutations in culture, and both their genotype and phenotype are different from those observed in native human cancer cells. ${ }^{1}$ Moreover, in vitro studies with cell lines do not reproduce the interactions with the prostate microenvironment, which is critical for PCa development and progression. Xenografts and genetically engineered mice represent improved models but have inherent advantages and limitations regarding mimicking PCa in humans. These models have advanced our understanding of PCa behavior, but differences between species and donors may lead to difficulties in interpreting the results. In addition, their deviation from human physiology could result in inaccurate preclinical assessment. ${ }^{2}$ To address these limitations, the use of ex vivo culture of human tissues has been described for different types of cancers. ${ }^{3-6}$ Culture of tissue slices is a good representative model of the

Supported by the Region Center Val de Loire, Project ReSCaP Canceropole Grand Ouest, France.

Disclosures: None declared. 
in vivo situation in patients because it maintains both cancer cell heterogeneity and an intact microenvironment, which allows stromal-epithelial interactions. In the past, in vitro maintenance of prostate tissues has been challenged by rapid degradation and poor survival of epithelial cells. ${ }^{7,8}$ However, the use of precision-cut slicing has led to more reproducible models, which have been reported by a few teams in preclinical studies. ${ }^{9-12}$ However, in these previous studies, the culture conditions did not take into account the specific variations related to the tumor microenvironment.

Essential factors such as hypoxia and calcium signaling have been shown to influence tumor progression and resistance to treatment. Hypoxia is a feature characteristic of most solid tumors, is a major contributor to cancer progression and resistance to treatment, and it exerts its effects mainly through the process of epithelial to mesenchymal transition (EMT). ${ }^{13-15}$ Prostate tumors are known to grow under a hypoxic microenvironment, which comprises oxygen concentrations lower than those found in the normal prostate. ${ }^{16,17}$ In addition, $\mathrm{Ca}^{2+}$ signaling associated with variations in extracellular $\mathrm{Ca}^{2+}$ concentrations has been shown to play a major role in the regulation of cellular processes leading to tumor progression, including proliferation, migration, and apoptosis. ${ }^{18-20}$ Extracellular calcium concentrations in the tumor microenvironment can reach levels between 8 and $40 \mathrm{mmol} / \mathrm{L}$, and it has been implicated in the development of bone metastasis. ${ }^{21}$ Moreover, altered expression of specific $\mathrm{Ca}^{2+}$ channels and pumps are characteristic features of most cancers including $\mathrm{PCa}{ }^{22}$ Therefore, the sensitivity of human cancer samples to extracellular calcium concentrations is a major element that needs to be assessed to show the functional value of an ex vivo prostate tissue model. Here, we describe a relevant and functional model of cultured benign and malignant human prostate tissues that preserves hypoxia and calcium sensitivity. The development of this model will allow a better analysis of the intrinsic properties of $\mathrm{PCa}$ and, possibly, the rapid and accurate identification of targeted cancer treatment.

\section{Materials and Methods}

\section{Patients and Tissues}

Prostatic tissue samples, including malignant and normal tissues, were obtained from 26 patients undergoing radical prostatectomy for clinically localized PCa. Tumors were classified according to the International Society of UroPathology (ISUP) in groups 1 to 5, and according to the pTNM stage as pT2 or pT3 (Table 1). Written informed consent was received from all patients.

\section{Preparation of Tissue Slices}

Radical prostatectomy specimens were obtained immediately after surgery, and 4- to 5-mm samples were dissected aseptically from the peripheral zone within the putative benign or
Table 1 Characteristics of Patients and Tissues

\begin{tabular}{ll}
\hline Characteristic & Value \\
\hline Age in years, median (range) & $67(51-76)$ \\
PSA, ng/mL (range) & $9.4(7-16)$ \\
pTNM, $n$ & \\
pT2 & 15 \\
PT3 & 11 \\
ISUP group, $n$ & \\
1 & 2 \\
2 & 11 \\
3 & 8 \\
$4-5$ & 5 \\
\hline
\end{tabular}

ISUP, International Society of UroPathology.

cancer areas (Figure 1). Tissue samples were glued against an agarose block (8\% agarose) that was stair-step cut and were immersed in RPMI medium supplemented with $10 \%$ fetal bovine serum and $1 \%$ penicillin-streptomycin. Briefly, the samples were cut immediately with a vibratome (Vibratome VT1200; Leica, Nanterre, France) at 200- to 300- $\mu$ m thickness, with a total number of slices ranging from 6 to 10 per case. Slices were used for either ex vivo culture and biomarker expression (15 patients) or for ex vivo culture and intracellular $\mathrm{Ca}^{2+}$ measurements (11 patients).

\section{Ex Vivo Culture of Prostate Tissue Slices}

Each slice was transferred into a 6-well cell culture plate containing Dulbecco's modified Eagle medium (Lonza, Levallois-Perret, France) supplemented with $10 \%$ fetal
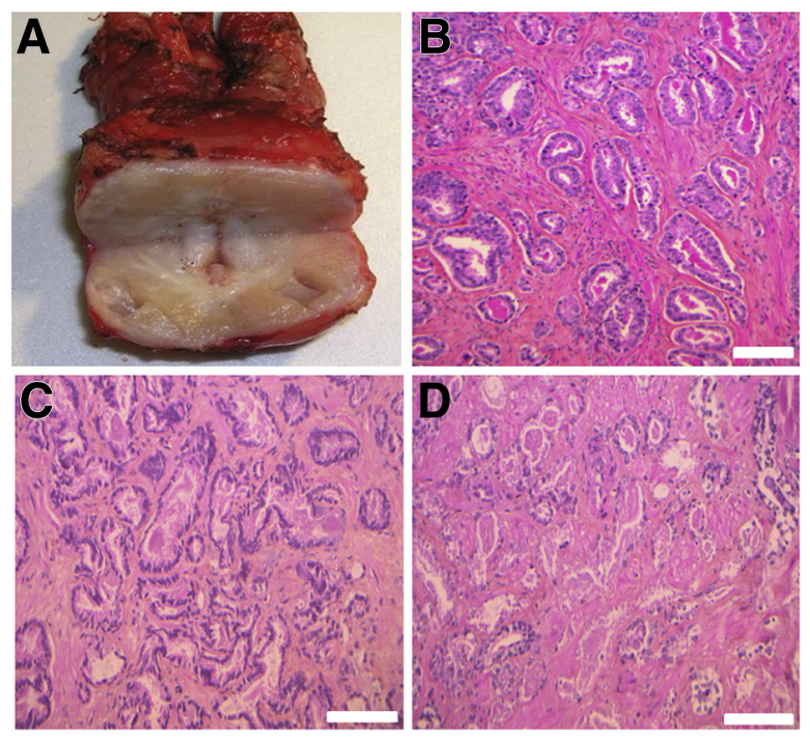

Figure 1 Tissue morphology and maintenance in culture. Radical prostatectomy specimen cut in a transverse plane. A: Tissue samples were taken from the peripheral zone in putative benign and cancer zones that were determined after palpation. B-D: Cancer samples from the same tumor before culture (B), after 5 days of culture with preservation of the tissue architecture and without necrosis (C), and after 7 days of culture, with areas of necrosis (D) (hematoxylin-eosin-saffron). Scale bars: $100 \mu \mathrm{m}$ (B-D). 
bovine serum and $1 \%$ penicillin-streptomycin, and incubated with or without $1 \mathrm{nmol} / \mathrm{L}$ dihydrotestosterone (DHT) (D073; Sigma Aldrich, St. Louis, MO). Slices were incubated in a $37^{\circ} \mathrm{C}$ humidified incubator with $5 \% \mathrm{CO}_{2}$. Medium was changed daily.

Hypoxia experiments were performed in a hypoxia chamber (SCI-tive; Alliance Bio Expertise, Guipry, France) in $1 \% \mathrm{O}_{2}$ at $37^{\circ} \mathrm{C}$ in a $5 \% \mathrm{CO}_{2}$ humidified environment. Slices first were placed in normoxia for 24 hours, to avoid additional stress after cutting, and then were incubated for 24 hours in a hypoxia environment. The medium was replaced every day.

Slices were cultured between 3 and 7 days after tissue collection. Analysis of sections after hematoxylin-eosin-saffron and immunohistochemical staining was performed at baseline (day 0), and then for day 1 (D1) to D3, or D1 to D7, depending on the number of slices available. In four cases the number of slices was sufficient to analyze the morphology from D0 to D7. An analysis of biomarker expression was performed in 15 cases at D0 and after 48 hours of culture. The effects of DHT deprivation and hypoxia were measured in 15 samples for DHT deprivation and in 5 samples for hypoxia at D2.

\section{Immunohistochemistry}

After fixation in $10 \%$ formalin, tissue slices were embedded in paraffin and sections were serially cut at $5 \mu \mathrm{m}$ with a microtome. Sections then were spread on a SuperFrost Plus slide (Fischer, Illkirch, France) and dried for 1 hour at $56^{\circ} \mathrm{C}$. One section was stained with hematoxylin-eosin-saffron for morphologic analysis. The other slides, used for immunostaining, were deparaffinized (histosol and alcohol baths), rehydrated, and heated in citrate buffer at $\mathrm{pH} 6$ (citric acid monohydrate and sodium citrate) for antigenic retrieval. After blocking for endogenous peroxidase with 3\% hydrogen peroxide, primary antibodies were incubated with the section. The panel of primary antibodies included p63 (clone 4A4, dilution 1/100, 30 min; Biocare, Concord, CA), p504S (clone 13H4, 1/200, 30 min; DakoCytomation, Glostrup, Denmark), androgen receptor (AR) (clone 1G3, 1/500, 30 min; Abnova), prostate specific antigen (PSA; clone BSB7,1/250, $30 \mathrm{~min}$; BioSB, Santa Barbara, CA), zinc finger E-box binding homeobox 1 (ZEB1; clone 4C4, 1/500, 1 h; Abnova), carbonic anhydrase IX (CAIX) (clone TH22, 1/100, 30 min; Novocastra, Newcastle upon Tyne, UK), ETS-related gene (EGR; EPR 3864, 1/1, 30 min; Roche France, Meylan, France), and the proliferation marker Ki-67 (clone 39-9, 1/50, $30 \mathrm{~min}$; DakoCytomation). Immunohistochemistry was performed with either the automated BenchMark XT slide stainer (Ventana Medical Systems, Inc., Orovalley, AZ) using the OptiView Detection Kit (Ventana Medical Systems, Inc.) or manually (for Zeb1) using the streptavidin-biotin-peroxidase method with diaminobenzidine as the chromogen (Kit LSAB; DakoCytomation). Slides were finally counterstained with hematoxylin. Negative controls were obtained after omission of the primary antibody or incubation with an irrelevant antibody.

\section{Scoring of Antibody Staining}

Staining for p63, p504, CAIX, and PSA was scored as positive or negative, and staining for AR, ERG, and Zeb1 was evaluated as follows: -, no stained cells; +, focal positivity; ++ , diffuse positivity. The number of $\mathrm{Ki}-67-$ positive cells was expressed as the percentage of total epithelial cells.

\section{Intracellular $\mathrm{Ca}^{2+}$ Measurements}

Intracellular $\mathrm{Ca}^{2+}$ measurements were performed on tissue slices after 48 hours of ex vivo culture. Intracellular calcium variations were assessed with the rhod-2 calcium-sensitive dye. All of the next steps were performed in darkness. Briefly, human prostate slices were incubated for 30 minutes with $5 \mu \mathrm{mol} / \mathrm{L}$ Rhod-2 acetoxymethyl (AM) at room temperature. Slices were placed in the bath of a Macro Zoom System Microscope MVX10 (Olympus France, Rungis, France) and were washed for 20 minutes with a physiological saline solution before experiments, using a pump in an open circuit. The dye was excited at $545 \pm 20 \mathrm{~nm}$ with light-emitting diode (LED) illumination (CoolLED pE-300
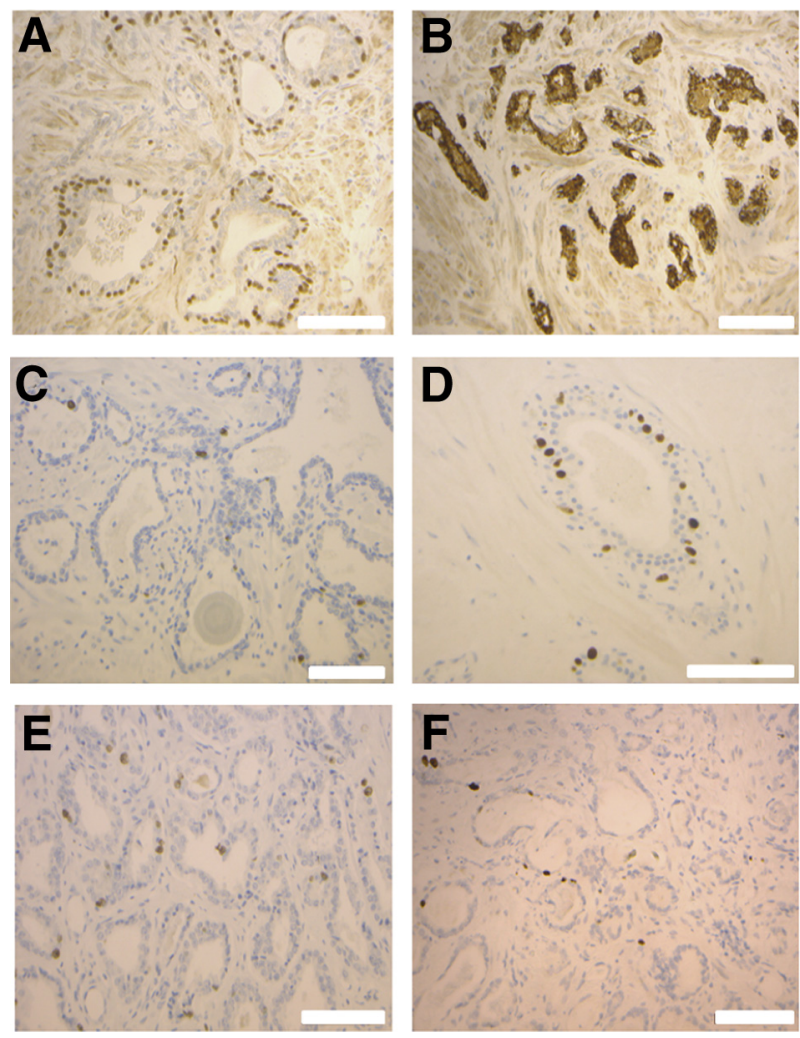

Figure 2 Biomarker expression in samples cultured for 2 days under normoxic conditions. $\mathbf{A}$ and $\mathbf{B}$ : Normal glands show preservation of the p63positive basal cell layer (A), whereas cancer cells are positive for p504s (B). C and D: When compared with the baseline status (D0) (C), the proliferation rate (determined by Ki-67 staining) was increased in the basal cell layer of normal glands, with several positive cells per gland (D). $\mathbf{E}$ and $\mathbf{F}$ : In cancer cells, the proliferation rate remained identical after culture $(\mathbf{F})$, when compared with D0 (E), with less than $3 \%$ positive cells. Scale bars: $100 \mu \mathrm{m}$ $(\mathbf{A}-\mathbf{F})$. 
white; CollLED, Andover, UK), and fluorescence was collected at $605 \pm 40 \mathrm{~nm}$ with a sCMOS Zyla 4.2 PLUS camera (Oxford Instruments, Abingdon-on-Thames, UK) and a Macro Zoom System Microscope MVX10. The image acquisition rate was $0.1 \mathrm{~Hz}$ and exposure time was $600 \mathrm{~ms}$. For each measurement, human prostate samples were incubated with $2 \mathrm{mmol} / \mathrm{L} \mathrm{CaCl}_{2}$ in physiological saline solution. After a stabilization time, $\mathrm{CaCl}_{2}$ was added to the incubation bath to reach a final concentration of $5 \mathrm{mmol} / \mathrm{L}$ $\mathrm{Ca}^{2+}$. Analyses were performed with ImageJ analysis software version 1.52a (NIH, Bethesda, MD; https://imagej.nih. gov/ij). The $\mathrm{Ca}^{2+}$ fluorescence signal was normalized with the initial fluorescence signal obtained at $2 \mathrm{mmol} / \mathrm{L} \mathrm{CaCl}_{2}$ to correct for differences in dye loading across the preparation. Variations of the $\mathrm{Ca}^{2+}$ signal then were determined by calculating the difference between the basal value, at $2 \mathrm{mmol} / \mathrm{L} \mathrm{CaCl}{ }_{2}$, and the maximal $\mathrm{Ca}^{2+}$ value after addition of $5 \mathrm{mmol} / \mathrm{L} \mathrm{CaCl}_{2}$.

\section{Statistical Analysis}

Statistical analyses were performed with StatView software version 5.0 (Abacus Concepts, Berkeley, CA). Comparison between groups was performed using the $\chi^{2}$ test for categoric data and the nonparametric $U$-test for continuous data. Paired data were analyzed using the nonparametric Wilcoxon test.

\section{Results}

\section{Tissue Morphology}

Tissue morphology after culture under normoxic conditions was compared with the morphology of native tissues (baseline, at D0). For all of the tested tissues, the architecture was preserved in both benign and malignant tissues from D1 to D5. No necrosis was observed between D1 and D5, but at D6 tissue samples started showing some necrosis foci, and at D7 necrosis was extensive (Figure 1).

\section{Biomarker Expression}

The expression of biomarkers after culture under normoxic conditions for 2 days was compared with native tissues (baseline, at D0). Biomarkers included markers of normal basal epithelial cells (p63), prostate cancer cells (p504S), proliferation (Ki-67), EMT (Zeb1 transcription factor), hypoxia (CAIX), and the fusion gene TMPRSS2-ERG product (ERG).
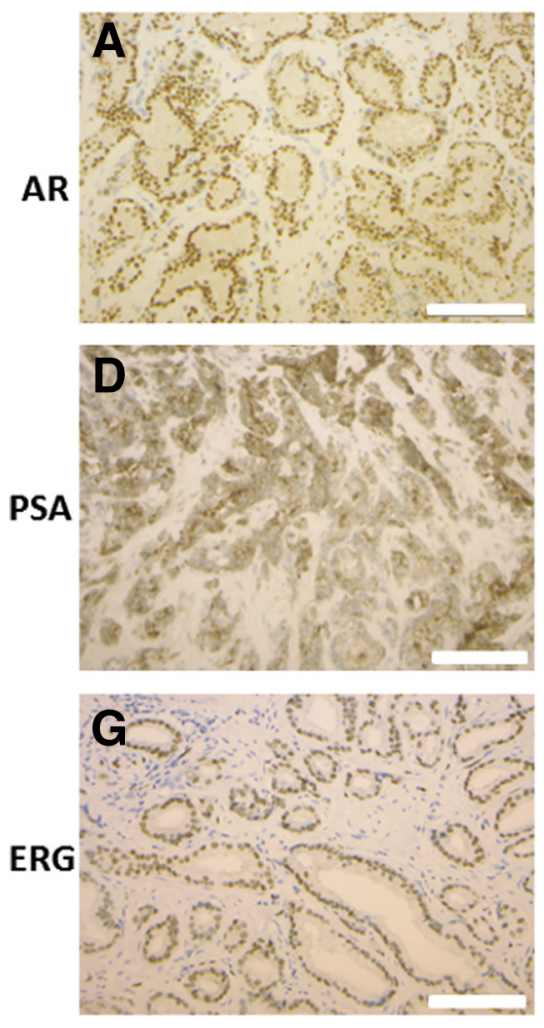

D0
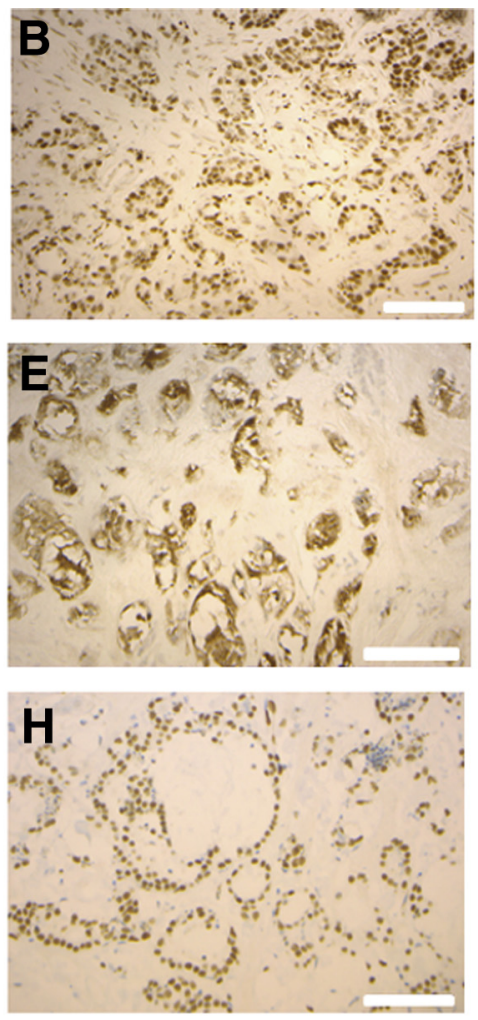

D2 with DHT
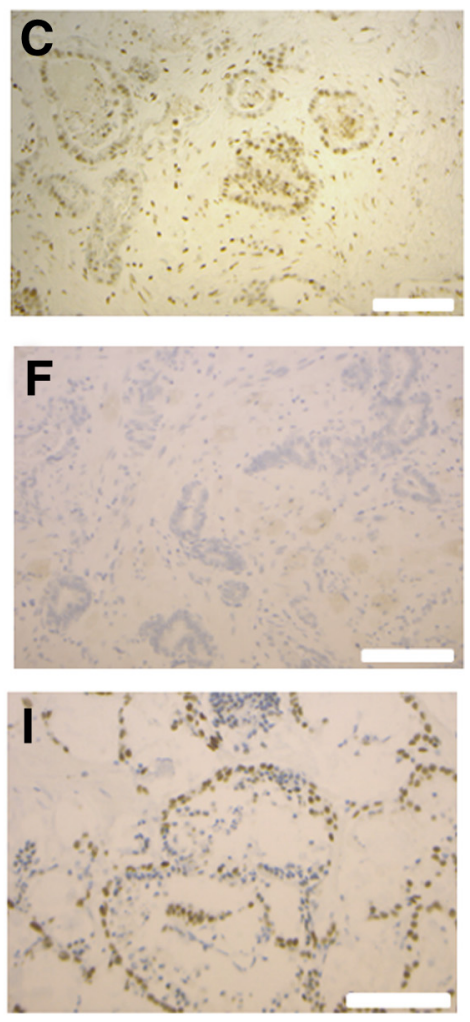

D2 without DHT

Figure 3 Expression of androgen receptor (AR) $(\mathbf{A}-\mathbf{C})$, PSA $(\mathbf{D}-\mathbf{F})$, and ERG $(\mathbf{G}-\mathbf{I})$ in native tumors (D0), and cancer samples cultured for 2 days with or without dihydrotestosterone (DHT). In the presence of DHT, AR, prostate specific antigen (PSA), and ETS-related gene (ERG) expression in cancer cells (B, E, and $\mathbf{H}$ ) remains identical to that observed at baseline (DO) (A, D, and $\mathbf{G})$. In contrast, DHT ablation lead to decreased expression of AR (C) and ERG (I), and PSA staining was abolished (F). Scale bars: $100 \mu \mathrm{m}(\mathbf{A}-\mathbf{I})$. 
In nontumor tissues $(n=7)$, all benign glands showed preservation of the p63-positive basal cell layer (Figure 2A). In malignant tissue slices $(n=8)$, cancer cells expressed p504S (Figure 2B) in all cases, as observed in the native tumors.

In normal glands at D0, proliferation was limited and restricted to basal cells (median, $1 \% \mathrm{Ki}-67-$ positive cells in the epithelial compartment; range, $1 \%$ to $3 \%$ ). Basal cell proliferation increased after culture (median, $8 \% \mathrm{Ki}$ 67-positive cells in the epithelial compartment; range, $6 \%$ to 15\%) when compared with the baseline status $(P<0.02$, Mann-Whitney test) (Figure 2, C and D). In tumor tissues, the proliferation rate was identical after culture (median, 3\%; range, $2 \%$ to $5 \%$ ) when compared with native tumors at D0 (median, 3\%; range, $1 \%$ to $4 \%)(P=0.8)$ (Figure 2, E and F).

At baseline (D0), prostate cancer cells expressed ERG in four of eight cases (50\%). In all positive cases, ERG expression was preserved in the cultured slices, and ERGnegative tumors remained negative after tissue culture. ERG expression was negative in all normal glands at both baseline and after culture. PSA was expressed in all cases of both normal and cancer tissues both before and after culture. In normal tissues at D0, epithelial cells were negative for the Zeb1 transcription factor and for CAIX expression. In cultured slices obtained from normal tissues (DHT-supplemented or not), CAIX staining remained negative, and focal expression of Zeb1 (rare positive epithelial cells) was observed in two of seven cases (without a significant difference compared with baseline, $P=0.4$ ). At D0, three of eight malignant samples showed focal Zeb1 expression in cancer cells, without CAIX staining. After culture under normoxic conditions, CAIX remained negative. Tumors with focal Zeb1 expression at baseline remained unchanged, whereas two of the five initially Zeb1-negative cases showed focal expression (without a significant difference compared with D0, $P=0.6$ ).

\section{Effects of DHT Deprivation}

In both benign and malignant glands, the absence of DHT in the medium led to decreased expression of AR (from diffuse to focal staining) and its target, the PSA gene product (from positive to negative staining), when compared with slices cultured in medium supplemented with DHT $(P=0.0001)$ (Figure 3, A-F). In ERG-positive tumors, DHT ablation was associated with decreased ERG levels in cancer cells (from diffuse to focal staining) $(P<0.03)$ (Figure 3, G-I).

\section{Hypoxia Effects}

Hypoxic conditions were tested with DHT supplementation for five samples (two benign and three malignant). In normoxia, benign samples were negative for Zeb1 and CAIX, and malignant samples were negative for CAIX (Figure 4A), and focally positive for Zeb1 (Figure 4C). When cultured under hypoxic conditions, all samples (benign and malignant) showed intense and diffuse

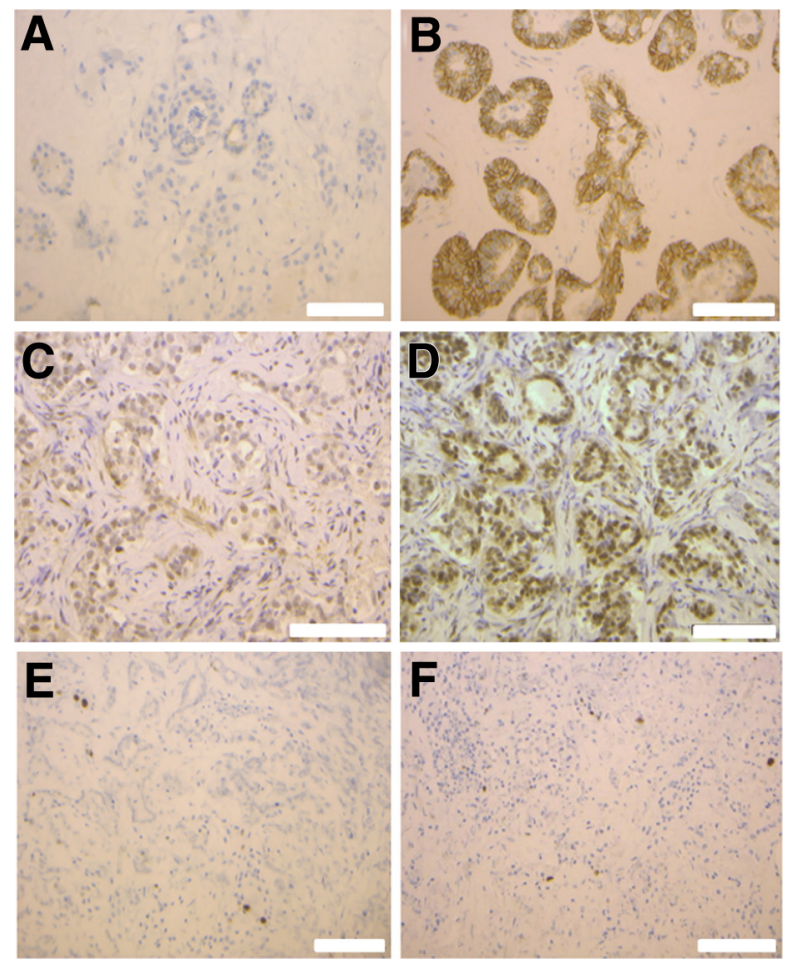

Figure 4 Effects of hypoxia on the expression of carbonic anhydrase IX (CAIX), zinc finger E-box binding homeobox 1 (Zeb1) transcription factor, and Ki-67. A and C: In cancer samples cultured in normoxic conditions, the hypoxia marker CAIX was negative (A), with negative to focal Zeb1 staining (C). B and D: After culture in hypoxia, cancer cells showed diffuse and membranous staining for CAIX (B), with diffuse nuclear staining for Zeb1 (D). E and F: In contrast, hypoxia does not modify cancer cell proliferation, with less than $3 \%$ of $\mathrm{Ki}-67$-positive cells in both normoxic $(\mathbf{E})$ and hypoxic (F) conditions. Scale bars: $100 \mu \mathrm{m}(\mathbf{A}-\mathbf{F})$.

membranous staining for CAIX $(P=0.001$ compared with normoxic conditions) (Figure 4B). In addition, in all cases, hypoxia also led to increased Zeb1 expression, with diffuse positive staining $(P=0.007$ compared with normoxic conditions) (Figure 4D). In contrast, hypoxia did not modify ERG expression in cancer cells as well as AR and PSA staining that remained positive (because hypoxic conditions were tested with DHT supplementation). Cancer cell proliferation also was identical in hypoxic compared with normoxic conditions, with a proliferation rate of less than 3\% (Figure 4, E and F).

\section{Ex Vivo Functional $\mathrm{Ca}^{2+}$ Imaging}

Finally, functional $\mathrm{Ca}^{2+}$ signaling was evaluated in prostate tissue slices obtained from tumor and adjacent nontumor tissues from 11 patients. This study was performed by measuring the variations in basal intracellular $\mathrm{Ca}^{2+}$ concentrations in response to variations of extracellular $\mathrm{Ca}^{2+}$ concentrations (from 2 to $5 \mathrm{mmol} / \mathrm{L}$ and 5 from $2 \mathrm{mmol} / \mathrm{L}$ ). Interestingly, as observed in the graphic trace, exposure to supraphysiological extracellular $\mathrm{Ca}^{2+}$ concentration $(5 \mathrm{mmol} / \mathrm{L}$ ) induced a robust and rapid increase of basal cytosolic $\mathrm{Ca}^{2+}$ concentration, resulting from a $\mathrm{Ca}^{2+}$ entry followed by a sustained plateau. 

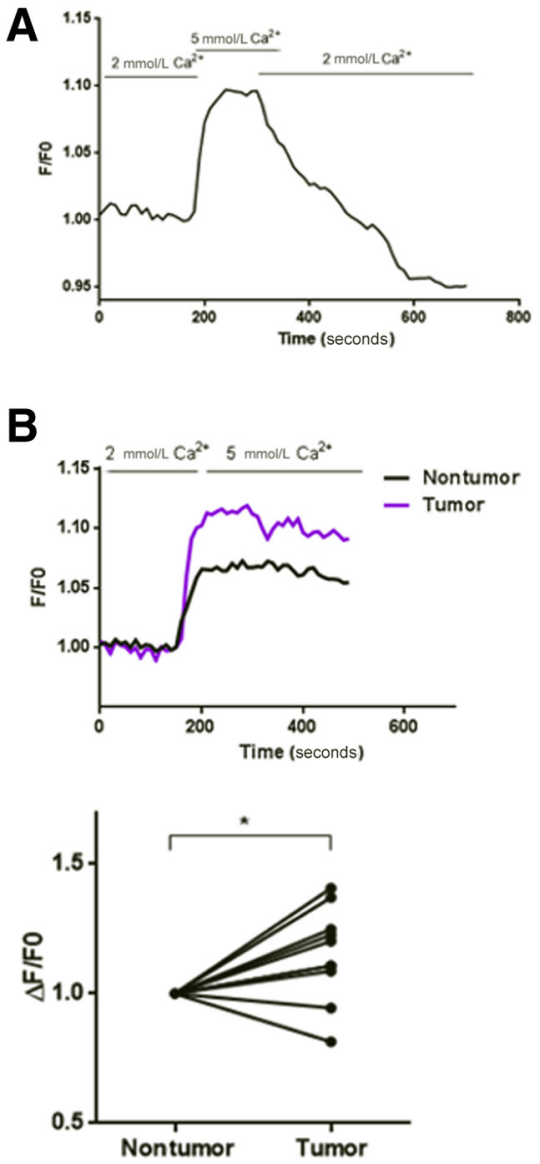
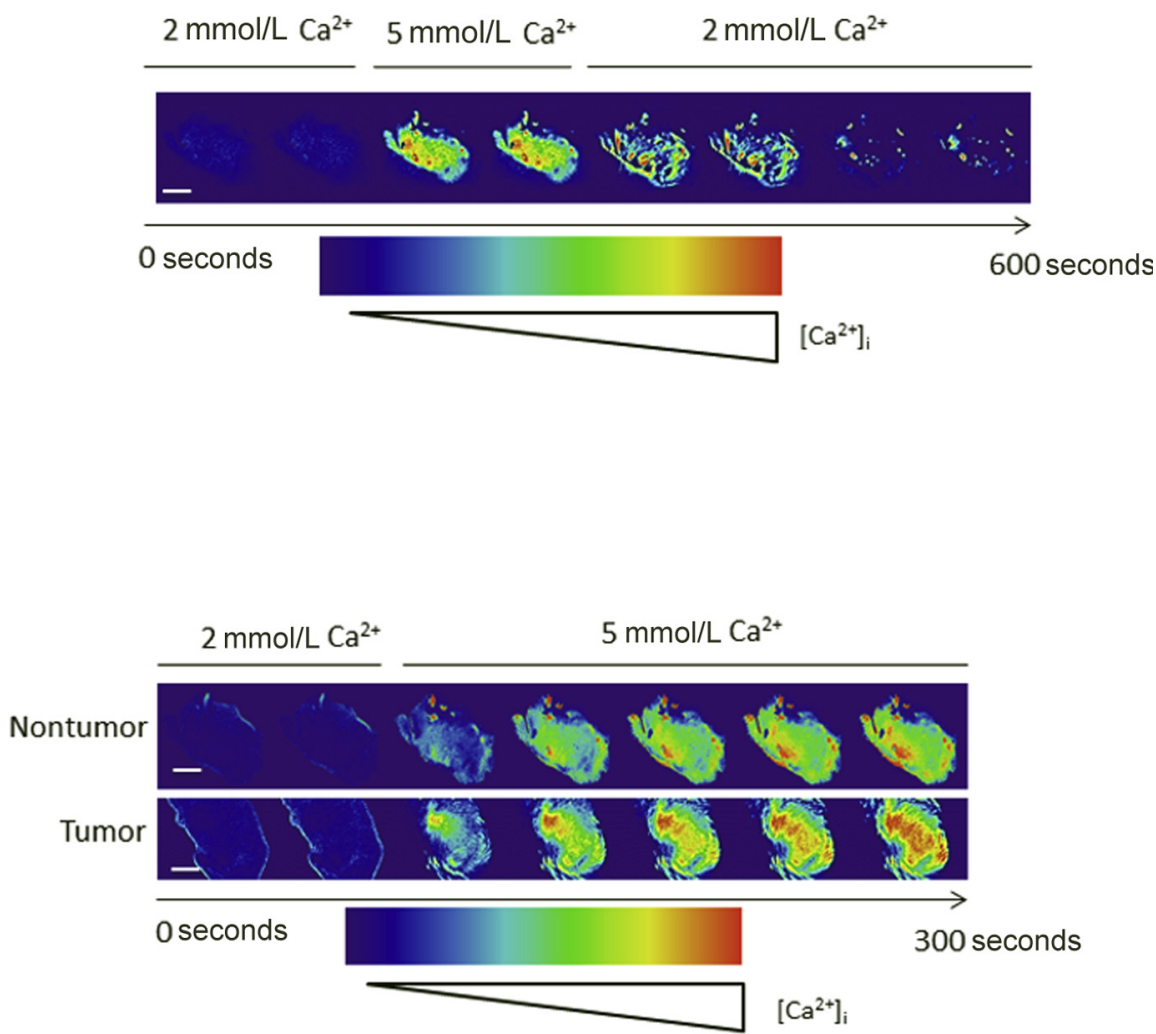

Figure 5 Variations in intracellular $\mathrm{Ca}^{2+}$ in human prostate slices. A: Exposure to supraphysiological extracellular $\mathrm{Ca}^{2+}$ concentrations (5 mmol/L) induced a robust and rapid $\mathrm{Ca}^{2+}$ entry followed by a sustained plateau and a slow return toward baseline by reducing extracellular $\mathrm{Ca}^{2+}$ concentration from 5 to 2 $\mathrm{mmol} / \mathrm{L}$. Real-time fluorescence images were collected every 80 seconds. B: Comparison of $\mathrm{Ca}^{2+}$ entry between tumor and adjacent nontumor prostate slices: in six of nine cases, $\mathrm{Ca}^{2+}$ entry was increased more in tumor compared with nontumor slices. Variations in $\mathrm{Ca}^{2+}$ signal were determined by calculating the differences between basal, at $2 \mathrm{mmol} / \mathrm{L} \mathrm{CaCl}$, and maximal $\mathrm{Ca}^{2+}$ value after addition of $5 \mathrm{mmol} / \mathrm{L} \mathrm{CaCl}_{2}(\Delta \mathrm{F} / \mathrm{FO})$. Real-time fluorescence images were collected every 10 seconds. $n=11$ patients. ${ }^{*} P<0.05$ (Wilcoxon). Scale bars: $2 \mathrm{~mm}$ (A and $\left.\mathbf{B}\right)$.

Interestingly, this increase could be reversed toward basal intracellular $\mathrm{Ca}^{2+}$ concentration when returning to a $2 \mathrm{mmol} / \mathrm{L}$ extracellular $\mathrm{Ca}^{2+}$ concentration (Figure 5A). The comparison of intracellular $\mathrm{Ca}^{2+}$ entry between tumor and adjacent nontumor prostate slices showed enhanced $\mathrm{Ca}^{2+}$ entry in malignant compared with benign tissues (for 9 of 11 cases) (Wilcoxon test, $P=0.014$ ) (Figure 5B). In tumor tissues, intracellular $\mathrm{Ca}^{2+}$ entry was greater in $\mathrm{PCa}$ from patients at high risk of recurrence (ISUP group 4 or 5 , and/or pT3) $(n=$ 5), compared with PCa from patients at low or intermediate risk of recurrence (International Society of UroPathology groups 1 to 3 , and pT2) $(n=6)(U$-test, $P=0.045)$ (Table 2$)$.

\section{Discussion}

This study showed that prostate tissue slices can be cultured for up to 5 days without necrosis with a preserved morphology and, more importantly, with unaffected physiological properties. This longevity of prostate tissue is obtained by using precision-cut slicing and daily replacement of medium complemented with DHT, and it is identical to that described previously. ${ }^{4}$ The expression of classic cell type markers was maintained, and they included p63 for basal cells, and AR and PSA for luminal cells. The cancer cell-associated marker p504S was present in all malignant tissue slices. Moreover, ERG expression was maintained after tissue culture in initially positive ERG tumors. Importantly, this finding is in contrast with a previous study that reported a loss of ERG expression. ${ }^{4}$ Because ERGpositive staining in prostate cancer cells is mediated by the action of androgens on the fusion gene TMPRSS2$E R G,{ }^{24}$ this result suggests a fully functional ARdependent signaling pathway in our model.

Table 2 Differences in Calcium Entry

\begin{tabular}{lll}
\hline $\begin{array}{l}\text { Risk of recurrence } \\
\left.\text { (d'Amico criteria }^{23}\right)\end{array}$ & Median $\Delta \mathrm{F} / \mathrm{F} 0$ (range) & $P(U$-test) \\
\hline Low & $1.08(0.81-1.25)$ & 0.045 \\
High & $1.30(1.11-2.78)$ & \\
\hline
\end{tabular}


Increased proliferation of basal cells was observed in benign tissue slices after culture when compared with native tissues (D0). This finding, which likely reflects increased glandular regeneration after culture, also was observed in a previous study, ${ }^{7}$ but not in another study. ${ }^{4}$ However, in malignant samples, the proliferation rate remained identical in cancer cells after culture, as previously described. ${ }^{4}$ Because one of the major potential interests of ex vivo models is the testing of therapeutic agents, the important point was to maintain an identical proliferation rate in cancer cells before and after culture.

When removing DHT from the medium, decreased levels of proteins encoded by androgen-regulated genes was observed. These proteins include AR, PSA, and ERG, in both malignant and benign tissues. This demonstration of androgen dependence has been reported in only one previous study, ${ }^{4}$ but without effect on ERG, and it is mandatory to reproduce ex vivo the biology of human PCa cells. In fact, the AR is a key molecule that drives both normal prostate development and PCa. To date, the study of androgen action in the human prostate has been hampered by the lack of adequate models because most PCa cell lines used for in vitro studies are generated from long-term culture in the absence of androgens. ${ }^{2}$ The present ex vivo model therefore would allow studying the physiological effects of androgens in either normal or malignant human prostate.

Some previous studies have reported the functionality of $\mathrm{PCa}$ organotypic culture. Vaira et $\mathrm{al}^{3}$ used this model to show the effects of targeting phosphatidylinositol 3-kinase signaling on cancer cell proliferation and apoptosis. Other reports have shown that cytotoxic drugs and ionizing radiation can induce DNA damage in ex vivo culture of $\mathrm{PCa}$ slices, ${ }^{9}$ and that inhibition of poly ADP-ribose polymerase 1 (PARP-1) can lead to AR signaling alterations. ${ }^{12}$ However, no previous studies to date have analyzed the ability of an ex vivo $\mathrm{PCa}$ model to respond to major signals of a cancer microenvironment, hypoxia, and extracellular calcium.

Because the $\mathrm{Ca}^{2+}$ signal is a phenomenon well suited for the rapid transfer of information from the microenvironment to cancer cells, ${ }^{22}$ the functional value of ex vivo cultures also could be reflected by the ability of tissue slices to respond to variations in extracellular $\mathrm{Ca}^{2+}$ concentrations. We have developed an ex vivo organotypic culture method that for the first time is suitable for the evaluation of live $\mathrm{Ca}^{2+}$ signaling in both tumor and adjacent nontumor samples. This method allowed us to follow the variations of basal intracellular $\mathrm{Ca}^{2+}$ concentration dynamics in human prostate slices resulting from $\mathrm{Ca}^{2+}$ entry from the extracellular side of the plasma membrane. The functionality of our model was attested by the variations of intracellular $\mathrm{Ca}^{2+}$ concentrations in response to variations in extracellular $\mathrm{Ca}^{2+}$. An increased $\mathrm{Ca}^{2+}$ entry in malignant compared with benign tissues also was shown. These data are in agreement with previous studies reporting alterations in $\mathrm{Ca}^{2+}$ homeostasis in cancer cells compared with normal cells. ${ }^{25}$ This type of $\mathrm{Ca}^{2+}$ entry already was described in breast cancer cell lines as constitutive $\mathrm{Ca}^{2+}$ entry. ${ }^{26}$ Here, we show for the first time the existence of a constitutive $\mathrm{Ca}^{2+}$ entry in $\mathrm{PCa}$ and, more interestingly, its differential level in tumor versus nontumor tissues. A greater intracellular $\mathrm{Ca}^{2+}$ entry also was found in tissues sampled from high-risk PCa compared with tumors with low or intermediate risk of recurrence, suggesting that $\mathrm{Ca}^{2+}$ entry may contribute to cancer progression. There is substantial evidence to suggest an important role for $\mathrm{Ca}^{2+}$ during tumorigenesis. Many mitogens and tumor growth factors elicit rapid increases of intracellular calcium. Furthermore, $\mathrm{Ca}^{2+}$ is involved in cellular events that regulate cell-cycle progression, cell migration, angiogenesis, and apoptosis in various cancer cells. ${ }^{27}$ Our findings raise the possibility that $\mathrm{PCa}$ cells might be particularly susceptible to novel anticancer agents that target $\mathrm{Ca}^{2+}$ homeostasis. Elucidating the nature of $\mathrm{Ca}^{2+}$ channels involved in dysregulated constitutive $\mathrm{Ca}^{2+}$ entry therefore is essential to better understand the mechanisms contributing to malignant transformation and cancer progression.

Hypoxia is a common phenomenon observed in solid tumors, including PCa. In human tumors, the effects of hypoxia largely are mediated by the hypoxia-inducible factors - transcription factors that induce the expression of several proteins including CAIX. ${ }^{28}$ Immunohistochemical staining of CAIX has been shown to be an endogenous surrogate marker for marked hypoxia in several types of solid tumors. ${ }^{29-31}$ Here, we show for the first time that hypoxic conditions could induce the expression of CAIX in ex vivo cultures of human prostate, even in benign tissues. In PCa, EMT induced by hypoxia is involved in the resistance to both radiotherapy and chemotherapeutic treatment. ${ }^{15,32}$ The key transcription factor of EMT, Zeb1, is associated in human $\mathrm{PCa}$ with cancer progression, metastases, and decreased survival. ${ }^{33}$ Here, we showed that, together with CAIX induction, hypoxia led to increased expression of Zeb1 in epithelial cells. Because EMT is a reversible process driven mostly by epigenetic mechanisms, ${ }^{34,35}$ the use of organotypic cultures therefore could represent a valuable preclinical model to study the effects of drugs targeting epigenetics, such as histone-modifying enzymes.

In conclusion, we report for the first time an ex vivo culture model of human PCa that remains sensitive to androgen ablation, hypoxia, and extracellular $\mathrm{Ca}^{2+}$. This model could be used to test therapeutic agents in viable tumor samples, thereby allowing the development of a precision medicine in clinical practice.

\section{References}

1. Toivanen R, Taylor RA, Pook DW, Ellem SJ, Risbridger GP: Breaking through a roadblock in prostate cancer research: an update on human model systems. J Steroid Biochem Mol Biol 2012, 131:122-131

2. Chauchereau A: Experimental models for the development of new medical treatments in prostate cancer. Eur J Cancer 2011, 47 Suppl 3: S200-S214

3. Vaira V, Fedele G, Pyne S, Fasoli E, Zadra G, Bailey D, Snyder E, Faversani A, Coggi G, Flavin R, Bosari S, Loda M: Preclinical model 
of organotypic culture for pharmacodynamic profiling of human tumors. Proc Natl Acad Sci U S A 2010, 107:8352-8356

4. Maund SL, Nolley R, Peehl DM: Optimization and comprehensive characterization of a faithful tissue culture model of the benign and malignant human prostate. Lab Invest 2014, 94:208-221

5. Carranza-Torres IE, Guzmán-Delgado NE, Coronado-Martínez C, Bañuelos-García JI, Viveros-Valdez E, Morán-Martínez J, CarranzaRosales P: Organotypic culture of breast tumor explants as a multicellular system for the screening of natural compounds with antineoplastic potential. Biomed Res Int 2015, 2015:618021

6. Koerfer J, Kallendrusch S, Merz F, Wittekind C, Kubick C, Kassahun WT, Schumacher G, Moebius C, Gaßler N, Schopow N, Geister D, Wiechmann V, Weimann A, Eckmann C, Aigner A, Bechmann I, Lordick F: Organotypic slice cultures of human gastric and esophagogastric junction cancer. Cancer Med 2016, 5:1444-1453

7. Papini S, Rosellini A, Campani D, DeMatteis A, Selli C, Revoltella RP: Selective growth of epithelial basal cells from human prostate in a threedimensional organ culture. Prostate 2004, 59:383-392

8. Bläuer M, Tammela TL, Ylikomi T: A novel tissue-slice culture model for non-malignant human prostate. Cell Tissue Res 2008, 332: 489-498

9. Jäämaa S, Af Hällström TM, Sankila A, Rantanen V, Koistinen H, Stenman UH, Zhang Z, Yang Z, De Marzo AM, Taari K, Ruutu M, Andersson LC, Laiho M: DNA damage recognition via activated ATM and 553 pathway in nonproliferating human prostate tissue. Cancer Res 2010, 70:8630-8641

10. Zhang Z, Yang Z, Jäämaa S, Liu H, Pellakuru LG, Iwata T, af Hällström TM, De Marzo AM, Laiho M: Differential epithelium DNA damage response to ATM and DNA-PK pathway inhibition in human prostate tissue culture. Cell Cycle 2011, 10:3545-3553

11. Centenera MM, Gillis JL, Hanson AR, Jindal S, Taylor RA, Risbridger GP, Sutherland PD, Scher HI, Raj GV, Knudsen KE, Yeadon T; Australian Prostate Cancer BioResource, Tilley WD, Butler LM: Evidence for efficacy of new Hsp90 inhibitors revealed by ex vivo culture of human prostate tumors. Clin Cancer Res 2012, 18: $3562-3570$

12. Schiewer MJ, Goodwin JF, Han S, Brenner JC, Augello MA, Dean JL, Liu F, Planck JL, Ravindranathan P, Chinnaiyan AM, McCue P, Gomella LG, Raj GV, Dicker AP, Brody JR, Pascal JM, Centenera MM, Butler LM, Tilley WD, Feng FY, Knudsen KE: Dual roles of PARP-1 promote cancer growth and progression. Cancer Discov 2012, 2:1134-1149

13. Byrne NM, Nesbitt H, Ming L, McKeown SR, Worthington J, McKenna DJ: Androgen deprivation in LNCaP prostate tumour xenografts induces vascular changes and hypoxic stress, resulting in promotion of epithelial-to-mesenchymal transition. Br J Cancer 2016, 114:659-668

14. Rankin EB, Giaccia AJ: Hypoxic control of metastasis. Science 2016, 352:175-180

15. Montanari M, Rossetti S, Cavaliere C, D'Aniello C, Malzone MG, Vanacore D, Di Franco R, La Mantia E, Iovane G, Piscitelli R, Muscariello R, Berretta M, Perdonà S, Muto P, Botti G, Bianchi AAM, Veneziani BM, Facchini G: Epithelial-mesenchymal transition in prostate cancer: an overview. Oncotarget 2017, 8: 35376-35389

16. Vaupel P, Höckel M, Mayer A: Detection and characterization of tumor hypoxia using pO2 histography. Antioxid Redox Signal 2007, 9: $1221-1235$
17. McKeown SR: Defining normoxia, physoxia and hypoxia in tumoursimplications for treatment response. Br J Radiol 2014, 87:20130676

18. Roderick HL, Cook SJ: Ca2+ signalling checkpoints in cancer: remodelling $\mathrm{Ca} 2+$ for cancer cell proliferation and survival. Nat Rev Cancer 2008, 8:361-375

19. Prevarskaya N, Skryma R, Shuba Y: Calcium in tumor metastasis: new roles for known actors. Nat Rev Cancer 2011, 11:609-618

20. Montheith GR, Prevarskaya N, Roberts-Thomson SJ: The calciumcancer signalling nexus. Nat Rev Cancer 2017, 17:367-380

21. Breuksch I, Weinert M, Brenner W: The role of extracellular calcium in bone metastasis. J Bone Oncol 2016, 5:143-145

22. Montheith GR, Davis FM, Roberts-Thomson SJ: Calcium channels and pumps in cancer: changes and consequences. J Biol Chem 2012, 287:31666-31673

23. Hernandez DJ, Nielsen ME, Han M, Partin AW: Contemporary evaluation of the D'amico risk classification of prostate cancer. Urology 2007, 70:931-935

24. Kumar-Sinha C, Tomlins SA, Chinnaiyan AM: Recurrent gene fusions in prostate cancer. Nat Rev Cancer 2008, 8:497-511

25. Stewart TA, Yapa KT, Monteith GR: Altered calcium signaling in cancer cells. Biochim Biophys Acta 2015, 1848:2502-2511

26. Chantôme A, Potier-Cartereau M, Clarysse L, Fromont G, Marionneau-Lambot $S$, Guéguinou $M$, Pagès JC, Collin $C$, Oullier $T$, Girault A, Arbion F, Haelters JP, Jaffrès PA, Pinault M, Besson P, Joulin V, Bougnoux P, Vandier C: Pivotal role of the lipid Raft SK3Orail complex in human cancer cell migration and bone metastases. Cancer Res 2013, 73:4852-4861

27. Clapham DE: Calcium signaling. Cell 2007, 131:1047-1058

28. Balamurugan K: HIF-1 at the crossroads of hypoxia, inflammation, and cancer. Int J Cancer 2016, 138:1058-1066

29. Russell J, Carlin S, Burke SA, Wen B, Yang KM, Ling CC: Immunohistochemical detection of changes in tumor hypoxia. Int J Radiat Oncol Biol Phys 2009, 73:1177-1186

30. Forker L, Gaunt P, Sioletic S, Shenjere P, Potter R, Roberts D, Irlam J, Valentine H, Hughes D, Hughes A, Billingham L, Grimer R, Seddon B, Choudhury A, Robinson M, West CML: The hypoxia marker CAIX is prognostic in the UK phase III VorteX-Biobank cohort: an important resource for translational research in soft tissue sarcoma. Br J Cancer 2018, 118:698-704

31. Ambrosio MR, Di Serio C, Danza G, Rocca BJ, Ginori A, Prudovsky I, Marchionni N, Del Vecchio MT, Tarantini F: Carbonic anhydrase IX is a marker of hypoxia and correlates with higher Gleason scores and ISUP grading in prostate cancer. Diagn Pathol 2016, 11:45

32. Stark TW, Hensley PJ, Spear A, Pu H, Strup SS, Kyprianou N: Predictive value of epithelial-mesenchymal-transition (EMT) signature and PARP-1 in prostate cancer radioresistance. Prostate 2017, 77: $1583-1591$

33. Figiel S, Vasseur C, Bruyere F, Rozet F, Maheo K, Fromont G: Clinical significance of epithelial-mesenchymal transition markers in prostate cancer. Hum Pathol 2017, 61:26-32

34. Wang JQ, Wu KJ: Epigenetic regulation of epithelial-mesenchymal transition by hypoxia in cancer: targets and therapy. Curr Pharm Des 2015, 21:1272-1278

35. Lee JY, Kong G: Roles and epigenetic regulation of epithelialmesenchymal transition and its transcription factors in cancer initiation and progression. Cell Mol Life Sci 2016, 73:4643-4660 